\title{
Sketch-It-Up! Demo
}

\author{
Bulut Karakaya, Camilo Garcia, Daniel Rodriguez, Manoj Nityanandam, \\ Nadia Labeikovsky, and Theyab Al Tamimi \\ 700 Technology Drive, Pittsburgh, PA - 15219, United States of America \\ contactasketch-it-up.com
}

Keywords: Ideation, Risk free innovation.

\section{Description}

Every creative project needs to have an ideation process. A good ideation process relies on a simple yet effective way of putting ideas on the table and sorting through them, also discarding them easily if necessary. However, for this ideation to be successful it has to be a process in which cheap and simple ways of exploring ideas are used along with tools that are readily accessible to everyone in the field to use.

It is known that the video game industry is mostly a production-oriented industry. This emphasis on production has neglected research into the ideation process necessary in a field of this nature. Very little has been taken into account regarding new ideas of gameplay, rather the focus is more on the aesthetics of the game. This means that the gameplay of many games of the same genre is identical, except for the background story and characters. This phenomenon is linked to the rapid growth of the industry and the vast sums of money that it is needed in order to produce a modern game.

We feel that this is a problem that should be addressed and solved, and for this we have suggested a tool in which ideas can be "sketched" in minutes with a whole team of designers and developers.

This tool is called Sketch-it-up!, It is an extension of the GameSketching system conceived by Dr. John Buchanan and his team in 2007. It is not just a software tool or a solution to a particular problem, but rather a set of processes and technologies that allow game development teams to explore and communicate ideas. Sketch-it-up! builds on the previous GameSketching system making it more accessible, whether the users are gamers, programmers, designers or artists.

Sketch-it-up! is a process that should happen in parallel to ideation, brainstorming and tools creation in games. Not to be confused with prototyping, which would require having game ready assets, the concept of sketching relates to hasty executions of ideas without using game ready assets or code. This would enable focused conversation between members of a team about the bare bones of a game, the essentials, the pros and cons of the idea itself.

Up to this day this ideation process was done through paper sketching and similar contraptions. Our tool is different because it is interactive in such a way that every member of a team can control different aspects of the sketch. It also reduces the confusion on writing and erasing things in the same paper at once. Different views are 
also provided so that the idea can be expressed from different angles and different game genres can be sketched. It can be shared and worked upon in real time on any network like ad-hoc, LAN or internet which is a huge advantage over paper sketching.

Sketch-it-up! is built on a Client/Server architecture. We refer to the Clients as puppeteers, and these puppeteers are connected to a main server. The puppeteer and server then load up the same world and each time an object is added by a puppeteer, it appears throughout the world. To avoid confusion, all requests to add/modify/move characters go through the controller and are granted only if those objects belong to that particular puppeteer. Each of the puppeteers has his own camera view to facilitate better maneuvering of his puppets.

Sketch-It-Up! is built on Disney's Panda3D engine because of its simple licensing policy and rapid development cycle due to the use of Python. Sketch-It-Up! is preloaded with a number of assets that can be used to sketch many types of games. But if needed, assets can be created in Maya, 3D Studio Max or Blender and imported into the tool.

To make a tool that the industry really needs, we presented it to game companies and got their feedback. We also constantly tested the tool ourselves to fix bugs and add features. This led us to make the current, robust tool that we have now.

However, in making this tool, we found that it has its limitations, as not every question or every genre can be sketched. But we see that features can be born during a sketch thanks to the spontaneity and improvisation of the puppeteers doing the sketch. Situations can be created in order to reinforce a game idea already implemented making that game unique even though its genre relies on a determined mechanic, such as fighting games like Street Fighter where we could sketch the layout of the fight, but not the fight itself.

We have found that sketching also improves the quality of ideas while saving time and money while enabling the team to have fun in the process.

\section{References}

1. Sketch-It-Up!, http: //www. sketch-it-up.com

2. Panda3D, http: //www. panda3d.org 